\title{
Translational Repression in Malaria Sporozoites
}

\author{
Oliver Turque ${ }^{1}$, Tiffany Tsao ${ }^{1}$, Thomas $\mathrm{Li}^{1}$ and Min Zhang ${ }^{1,2, *}$ \\ ${ }^{1}$ HIV and Malaria Vaccine Program, Aaron Diamond AIDS Research Center, Affiliate of The Rockefeller University, New York, NY, USA. \\ ${ }^{2}$ Department of Pathology, New York University School of Medicine, New York, NY, USA. \\ * Corresponding Author: \\ Min Zhang, E-mail: Dr.Min.Zhang@gmail.com
}

\begin{abstract}
Malaria is a mosquito-borne infectious disease of humans and other animals. It is caused by the parasitic protozoan, Plasmodium. Sporozoites, the infectious form of malaria parasites, are quiescent when they remain in the salivary glands of the Anopheles mosquito until transmission into a mammalian host. Metamorphosis of the dormant sporozoite to its active form in the liver stage requires transcriptional and translational regulations. Here, we summarize recent advances in the translational repression of gene expression in the malaria sporozoite. In sporozoites, many mRNAs that are required for liver stage development are translationally repressed. Phosphorylation of eukaryotic Initiation Factor $2 \alpha$ (elF2 $\alpha$ ) leads to a global translational repression in sporozoites. The elf2 $\alpha$ kinase, known as Upregulated in Infectious Sporozoite 1 (UIS1), is dominant in the sporozoite. The elf2 $\alpha$ phosphatase, UIS2, is translationally repressed by the Pumilio protein Puf2. This translational repression is alleviated when sporozoites are delivered into the mammalian host.
\end{abstract}

Plasmodium sporozoites are quiescent for several weeks in mosquito salivary glands while maintaining their infectivity. In stark contrast to the parasite's continuous transcriptional changes in the liver (pre-erythrocytic) and blood (erythrocytic) stages in their vertebrate hosts, the sporozoite's transcriptional program remains unchanged during the long period of time in mosquito salivary glands. The transcriptional and translational profiles of sporozoites and pre-erythrocytic stage parasites display significant lags in protein abundance relative to mRNA abundance, due to a global translational repression caused by the phosphorylation of elF $2 \alpha$ in salivary gland sporozoites.

Eukaryotic Initiation Factor 2 (elF2), composed of $\alpha, \beta$, $\gamma$ subunits, recruits Met-tRNA and GTP to form a ternary complex that binds to ribosome, which initiates protein translation. GTP is then hydrolyzed, and the ribosome releases elF2-GDP as an inactive binary complex. EIF2B, a guanine nucleotide exchange factor, subsequently converts elF2-GDP to elF2-GTP, which is required for another round of translational initiation. Phosphorylation of elF $2 \alpha$ blocks the recycling of elF2-GDP to its translationally active elF2GTP form, thereby inhibiting global protein synthesis. The stalled mRNAs are then assembled as stress granules (messenger ribonucleoprotein complexes) in Plasmodium sporozoites. Activating Transcription Factor 4 (ATF4), a transcriptional activator of genes involved in the integrated stress response, is preferentially translated when elF $2 \alpha$ is phosphorylated in mammalian cells (Figure 1A). In Plasmodium, it is unknown if phosphorylation of elF $2 \alpha$ facilitates the preferential translation of select transcripts while repressing general translation initiation.

Plasmodium elF2 $\alpha$ kinase UIS1 (also termed elK1) phosphorylates elF $2 \alpha$ in salivary gland sporozoites. Uis genes are a subset of thirty genes that are transcriptionally upregulated when sporozoites differentiate from the mosquito midgut to salivary glands. The elF $2 \alpha$ phosphatase is UIS2 in Plasmodium salivary glands sporozoites, where the uis1 and uis2 mRNAs are most abundant among the uis transcripts. Plasmodium Pumilio protein Puf2 contains a sequence-specific RNA binding domain and serves as a translational repressor of specific mRNAs by binding to their 3'-untranslated region. Puf2 inhibits translation of uis2, consequently rendering UIS1 dominant. This leads to a prevalence of phosphorylated elF $2 \alpha$ and global protein repression in sporozoites (Figure 1B).

Many liver stage messages, such as uis2 and uis4, bind to Puf2 and are translationally repressed and stabilized in sporozoites (Figure 1). The transcriptome and morphology of puf2(-) sporozoites in $P$. berghei and $P$. yoelii rodent malaria, transform into early liver stages. The transcript of uis1 is degraded in the puf2(-) sporozoites. The phenotype

MICROREVIEW on: Zhang M, Mishra S, Sakthivel R, Fontoura BM, Nussenzweig V (2016). UIS2: A Unique Phosphatase Required for the Development of Plasmodium Liver Stages. PLoS Pathog 12(1): e1005370. doi:10.1371/journal.ppat.1005370. 
A

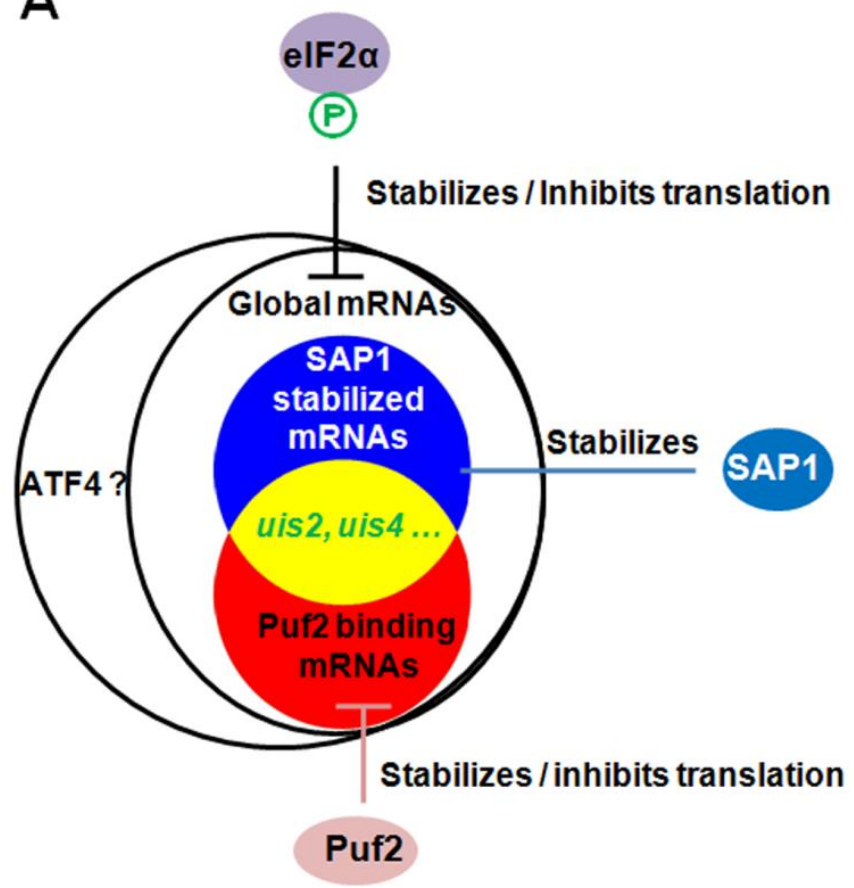

B
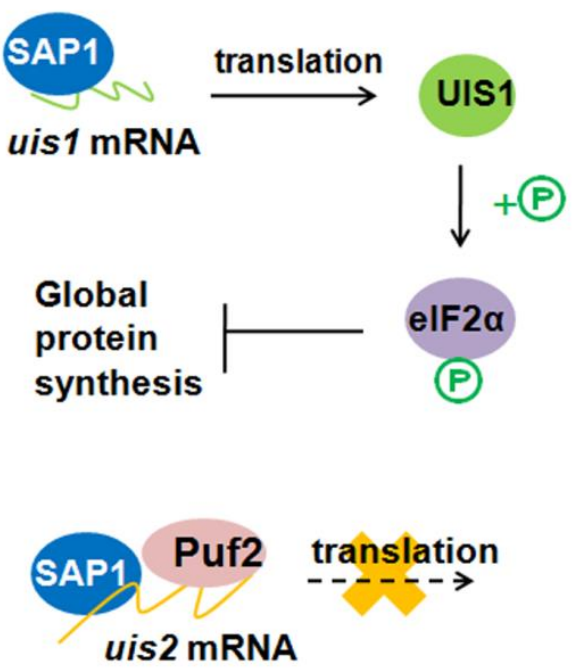

FIGURE 1: Model illustrating translational repression in malaria sporozoites. (A) Translational repression by elF2 $\alpha$ phosphorylation and Puf2. Puf2 stabilizes and inhibits translation of a group of mRNAs by binding to their 3' untranslated region. SAP1 stabilizes 38 uis mRNAs and nonuis mRNAs. EIF2 $\alpha$ phosphorylation inhibits global protein synthesis. The stalled mRNAs are assembled into RNA granules. (B) Regulation of elF2 $\alpha$ phosphorylation. Phosphorylation of elF $2 \alpha$ is controlled by the kinase UIS1 and the phosphatase UIS2. uis 1 and uis 2 transcripts are stabilized by SAP1, and the translation of uis 2 is repressed by Puf2. UIS1 is dominant, and causes dormancy in Plasmodium sporozoites.

of the elF2 $\alpha$ kinase uis1(-) is similar to that of puf2(-). The elF2 $\alpha$ phosphatase uis2(-) sporozoites do not transform into liver stages but maintain their crescent sporozoite shapes after delivery to host cells. The phenotype of uis2(-) is adverse to that of uis1(-) and puf2(-).

Sporozoite Asparagine-rich Protein 1 (SAP-1) is essential for the stability of a group of thirty-eight mRNA transcripts in $P$. yoelli salivary gland sporozoites. The transcripts of many uis genes are quickly degraded at their 3'ends, including uis1 and uis2. Additionally, in the sap1(-) sporozoite, some transcripts, including uis4, were shown to be quickly degraded at their 5 '-ends. Thus, in the absence of SAP-1, genes that are essential to liver-stage development of the infectious sporozoite follow the pattern in which the $3^{\prime}$ and $5^{\prime}$ ends of the mRNA molecule are sequentially degraded.

Since UIS2 is essential for liver-stage development of sporozoites, we explored its function. In mammalian cells, Protein Phosphatase 1 (PP1) with its cofactor GADD34 or CReP mediates the dephosphorylation of elF $2 \alpha-P$. However GADD34 and CReP are absent in Plasmodium. Our data show that Plasmodium PP1 does not dephosphorylate Plasmodium elF2 $\alpha-P$ in sporozoites. Rather, UIS2 is the elF $2 \alpha-P$ phosphatase that directly binds to its substrate elF2 $\alpha$-P. Our demonstration that UIS2 is a novel elF2 $\alpha-P$ phosphatase reveals another mechanism to control elF $2 \alpha$ dephosphorylation. Our findings also suggest that additional mechanisms have evolved to control elf $2 \alpha$ dephosphorylation in organisms that do not contain recognizable homologs of GADD34 or CReP.

UIS2 contains a conserved RVxF motif within its phosphatase domain (Figure 2). This motif is a putative PP1binding motif. Although PP1 is not an elF2 $\alpha$ phosphatase in sporozoites, we do not exclude the possibility that Plasmodium PP1 might be an elF $2 \alpha$ phosphatase in other stages of the malaria life cycle. An interesting hypothesis requiring further investigation is that Plasmodium PP1 is recruited by UIS2. The redundant eIF $2 \alpha$ phosphatase may result in rapid dephosphorylation of elF $2 \alpha$ during the sporozoite's transition from its latent form to its active form.

Plasmodium sporozoites remain latent in mosquito salivary glands until transmission to a host. The translational repression of liver stage messages such as uis2, uis3, and uis4 in sporozoites is correlated with the observation that these genes are not essential for the parasite's sporozoite stage development. Liver stage messengers start to translate by the alleviation of translational repression. The initiation factor, elF $2 \alpha$, is dephosphorylated in pre-erythrocytic stages where UIS2 is dominant. These parasites utilize continuous transcriptional changes for their pre-erythrocytic and erythrocytic stage development.

$P$. vivax infects humans and undergoes an especially dangerous type of latent morphology in the liver. Hypnozoites remain dormant in the liver of their host until they eventually cause a relapse of malaria. Though the transcriptional and translational profiles of hypnozoites are 


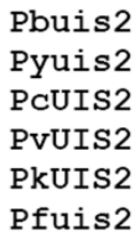

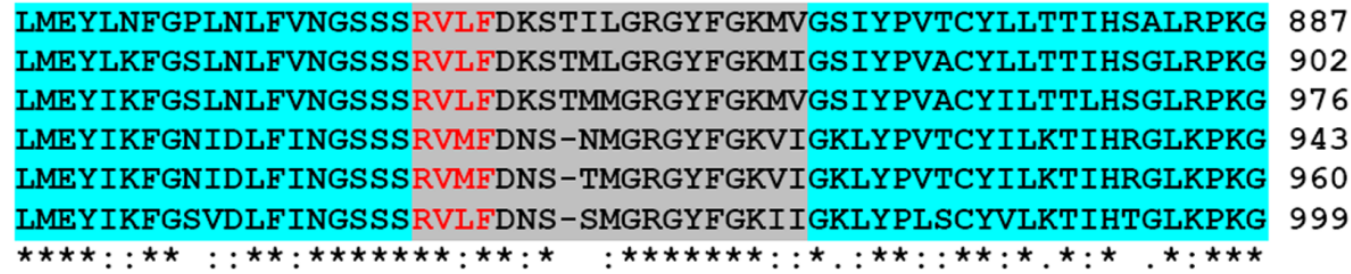

FIGURE 2: The RVxF motif in UIS2 phosphatase domain. $P$. berghei UIS2 (1321 amino acids) contains a phosphatase domain (roughly residues 535-1054). The amino acids around RVxF motif in UIS proteins from P. berghei, $P$. yoelii, P. chabaudi, $P$. vivax, $P$. knowlesi, and $P$. falciparum are aligned. The cyan area shows the conserved phosphatase domain and the gray region shows the insertion in UIS2 relative to 1 UTE (the best matching phosphatase in Protein Data Bank).

unknown, we speculate that translation is similarly repressed in hypnozoites, and that their latency is associated with elF $2 \alpha$ phosphorylation. One possibility is that UIS2 remains translationally repressed in hypnozoites until a signal (currently unknown) reactivates them.

\section{ACKNOWLEDGMENTS}

This work was supported by National Institutes of Health (grant Al108592) and Sao Paulo Research Foundation (FAPESP, grant 12/50399-4).

\section{CONFLICT OF INTEREST}

The authors declare that no competing interest exists.

\section{COPYRIGHT}

(C) 2016 Turque et al. This is an open-access article released under the terms of the Creative Commons Attribution (CC BY) license, which allows the unrestricted use, distribution, and reproduction in any medium, provided the original author and source are acknowledged.

Please cite this article as: Oliver Turque, Tiffany Tsao, Thomas Li and Min Zhang (2016). Translational Repression in Malaria Sporozoites. Microbial Cell 3(5): 227-229. 